\title{
VSA-CubeBot: a modular variable stiffness platform for multiple degrees of freedom robots.
}

\author{
Manuel G. Catalano ${ }^{\dagger \ddagger}$, Giorgio Grioli ${ }^{\dagger}$, Manolo Garabini ${ }^{\dagger}$, Fabio Bonomo $^{\dagger}$, \\ Michele Mancini ${ }^{\dagger}$, Nikolaos Tsagarakis ${ }^{\ddagger}$ and Antonio Bicchi ${ }^{\dagger} \ddagger$
}

\begin{abstract}
We propose a prototype of a Variable Stiffness Actuator (VSA) conceived with low cost as its first goal. This approach was scarcely covered in past literature. Many recent works introduced a large number of actuators with adjustable stiffness, optimized for a wide set of applications. They cover a broad range of design possibilities, but their availability is still limited to small quantities. This work presents the design and implementation of a modular servo-VSA multi-unit system, called VSA-CubeBot. It offers a customizable platform for the realization and test of variable stiffness robotic structures with many degrees of freedom. We present solutions relative to the variable stiffness mechanism, embedded electronics, mechanical and electrical interconnections. Characteristics, both theoretic and experimental, of the single actuator are reported and, finally, five units are interconnected to form a single arm, to give an example of the many possible applications of this modular VSA actuation unit.

Index Terms-Physical Human-Robot Interaction, Performance, Variable Stiffness Mechanisms, Actuators, Robot, multi degree of freedoms robots, Modular Robots, Humanoid
\end{abstract}

\section{INTRODUCTION}

The development of robots that can operate outside a structured environment (e.g. factories) and can interact with unknown scenarios, people and even other robots is one of the main goals of Robotics. Developing suitable actuation systems for these robots is one of the most investigated topics, and the implementation of new electro-mechanical solutions, control strategies and sensor systems is the result of such research activity.

One fundamental aspect for robots which interact with external bodies is: the robotic system impedance management [1]. If active impedance control is one of the most studied and popular techniques [2], the passive approach also got a big diffusion, especially during the past ten years. This technique sprouted into systems able to change their stiffness [3] or damping [4] using dedicated mechanisms. During last ten years more than fifteen prototypes of one degree of freedom (DOF) systems have been developed and many publications investigated and demonstrated the effectiveness of this technology and its feasibility (e.g. [5], [6], for an exhaustive list see [7]).

Many works explored the theoretical solutions of control problems for multi-DOF systems and hint the many possible

\footnotetext{
$\dagger$ Centro Int. di Ricerca "E. Piaggio", Univ. of Pisa, 56126 Pisa, Italy

‡ Italian Inst. of Technologies, Adv. Robotics, 16163 Genova, Italy

\{manuel.catalano, giorgio.grioli,

manolo.garabini, fabio.bonomo,

bicchi\}@centropiaggio.unipi.it

nikos.tsagarakiseit.it

mancini.m.f@gmail.com
}

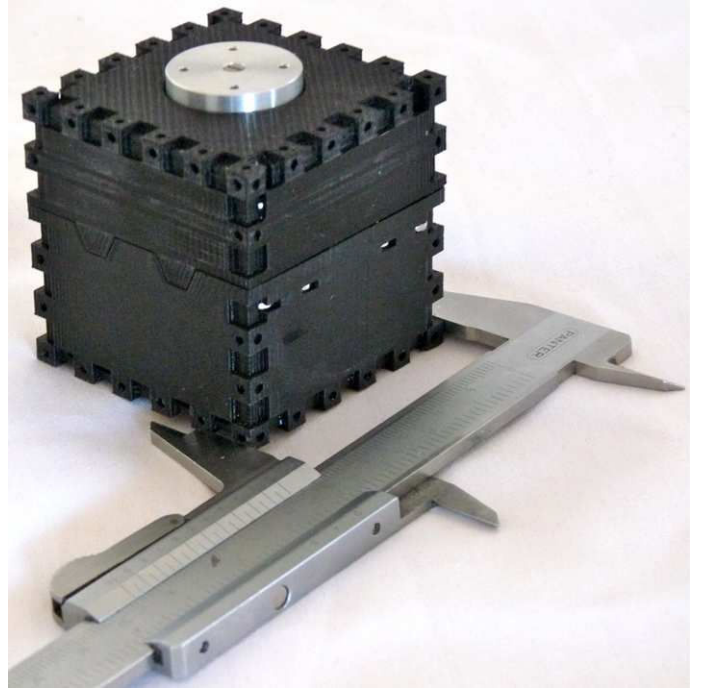

Fig. 1. VSA-Cube, a variable stiffness actuator for high modularity and low cost multi degrees of freedom robotic structures.

applications of this technology [8]. Nevertheless, considering the actual state of the art, experimental validations are difficult to be found.

One of the most relevant problems is, in fact, the relative novelty of this technology and the following absence of commercial prototypes. This limitation binds researchers to create their experimental set-up each time from scratch. The development of a new system requires time, money and specific know-how, and these issues are magnified for robotic structures with many degrees of freedom. Considering, as an example, the development of VSA-HD [9], costed about $10000 €$ for materials alone, it is easy to extrapolate what the cost of a multi-DOF system would be.

On the other hand the development of a system with traditional electriomagnetic actuation is easy because it is possible, today, to select the basic components from a big range of products, differing in price and performance. A typical example are the modular servo actuators by companies such as Schunk [10] or Harmonic Drive [11], which cover the high-end side of the market. More economic solution are available, as far as the specifications relax, reaching the relatively new robotic platforms developed for the edutainment market (e.g. Bioloid [12], Hitec [13] Lego-Mindstorm [14]), pushed also by competitions as Robocup [15]. All these platforms offer the possibility to realize multi-DOF systems at the cost of a reasonably low expense. Performance 
is obviously limited but, despite the fact that these systems can not be employed in industrial environments or big service robotics applications, they can still be profitably used for research and teaching activities, like in [16], [17], [18], [19].

The authors believe that an approach nearing the last examples could be useful for the development of variable impedance robots. This approach can improve the opportunity to validate and test these systems, for exploring various assembly possibilities and for interacting with different kind of environments.

This paper presents a novel prototype of servo VSA, the VSA-Cube, a picture of which is shown in Fig. 1. The main design considerations for this actuator are high modularity, small size, and low cost. This actuation unit is intended to be part of an advanced robotics kit. The basic idea is to present a system which is similar to a servo motor, so that assembly of any robotic system could only take into account performance and capabilities of the actuator as a whole: energy storage, stiffness range, stiffness settling time and stiffness/torque range. The first application implemented with the VSA-CubeBot platform is a humanoid upper body, shown in Fig. 10(a).

This article is structured as follows:

Sec. II describes the actuator, the mechanics and the electronic interface. Sec. III describes the model of the VSA Cube unit. Sec. IV describes the multi DOF platform, the proposed assembly and some experimental results. Finally in Sec. V the conclusion of the work are discussed.

\section{VSA-CUBE}

VSA-Cube is a variable stiffness servo actuator. The word "servo" conventionally describes an embedded system composed of a prime mover, a gearbox, a position sensor, an electronic board and the algorithms to control the output shaft position. A communication bus between the servo and the main high level controller is usually also embedded. In general the manufacturer develops the low level control electronics and the customer is provided only with the actuator output performance. Fig. 2 shows the typical datasheet of two servo motors, one from Schunk and one from Hitec Inc., where only information about output shaft position range, torque and velocity are published. Obviously this approach

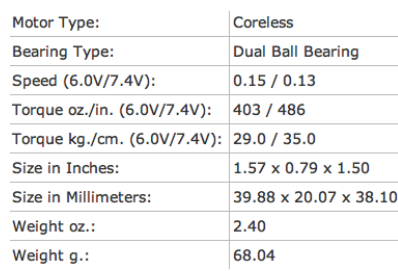

(a)

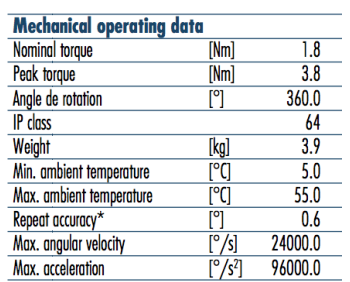

(b)
Fig. 2. Datasheets of two different servo actuator. An high end Schunk servo $(a)$ and a Hitec Inc. servo $(b)$. Only information about output performance are shown. Panel $(b)$ shows mechanical properties of the servo motor adopted in the VSA-Cube module.

is not sufficient for all applications but, in many cases, it is much more economic, fast and easy. The biggest advantage of this approach is that each servo motor is an independent subsystem and can be easily used in a big robotic framework exploiting this modularity.

The VSA-Cube embeds the features of a servo motor and, moreover, the possibility of adjusting the output shaft stiffness. Stiffness control is obtained, as for the output position, commanding the explicit reference. Similarly to a normal servo motor the user can employ the VSA-Cube relying only on the knowledge of its output performance.

The set of parameters needed to completely characterise a VSA is still an open issue. For a servo VSA the situation is simpler, especially from the user's point of view. A VSA can be seen as a system with a non linear transmission that transforms input torques and velocities of its prime movers into a set of four new variables relative to the output shaft: torque, velocity, stiffness and stiffness velocity. As discussed

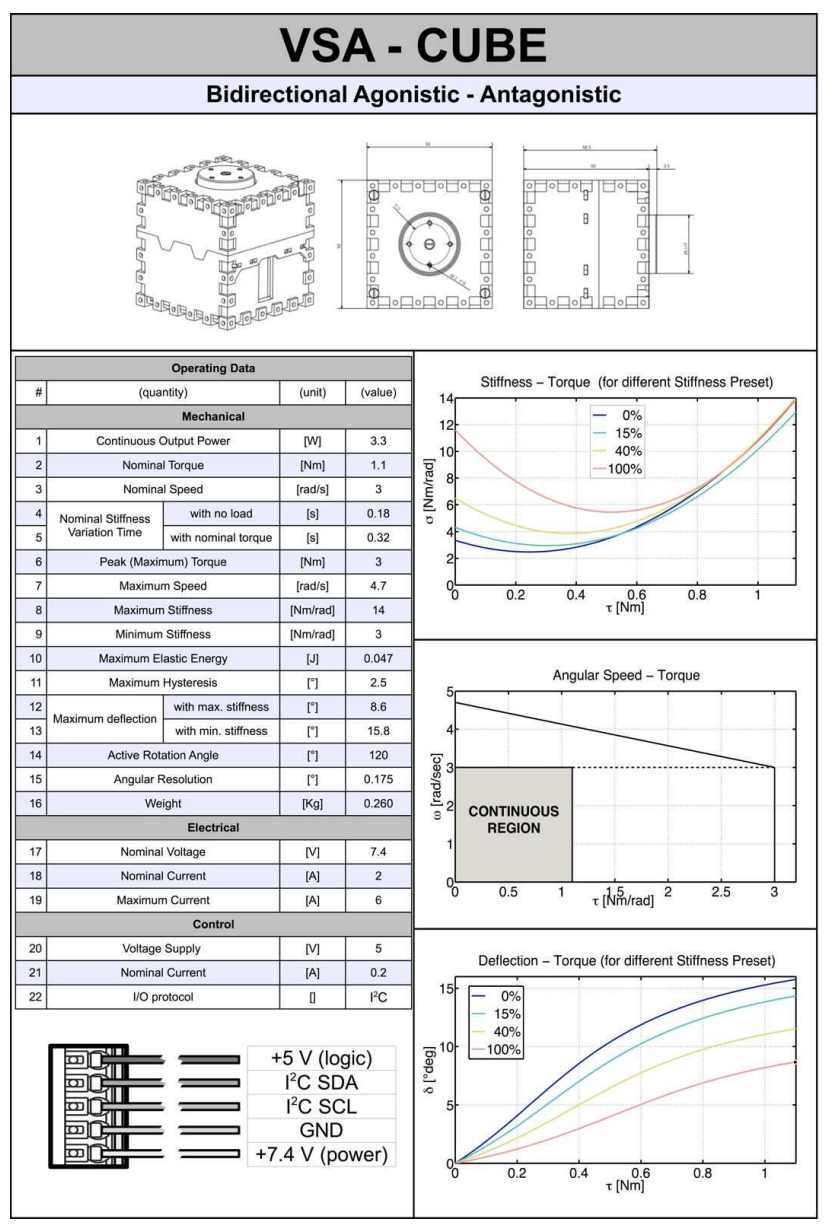

Fig. 3. The VSA-Cube datasheet. All of the data are obtained from experimental calibration, performed as in [9]. Curves on the right side are plotted for different percentages of the stiffness pre-set. Data refers to the continuous working region.

in [20] it is possible to map the torque $(\tau)$ / stiffness $(\sigma)$ and velocity $(\omega)$ / stiffness relations in a way similar to the torque / velocity relation of a traditional electric motor. Using the representation proposed in [20], [9] and [21], it is easy to see how the Performance Envelope Volume (PEV), presented in [20] (here showed in Fig. 4) describes 
the capability of an actuator to satisfy the requirements of a specific application. In Fig. 3 a complete data-sheet for the VSA-Cube is presented. Relations between torque, velocity and stiffness are reported in it using the projections of the three-dimensional volume, along with data about velocity and energy stored inside the system. Notice that the square

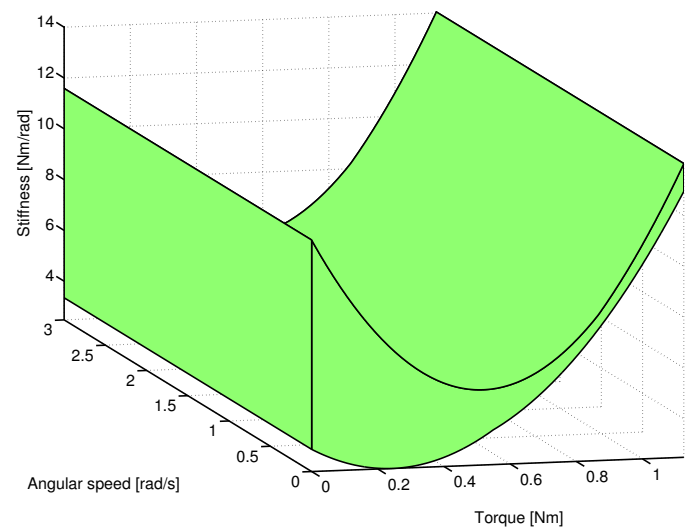

Fig. 4. Performance Envelope Volume (PEV) of one VSA-Cube unit as derived from experimental validation of the prototype. Plotted data represents the continuous working region.

characteristic plotted on the plane $\langle\tau, \omega\rangle$ is a consequence of the lowest layer of control, implementing a PID loop on the prime movers (Sec.II-B), this is also reflected in the PEV projection on the plane $\langle\omega, \sigma\rangle$.

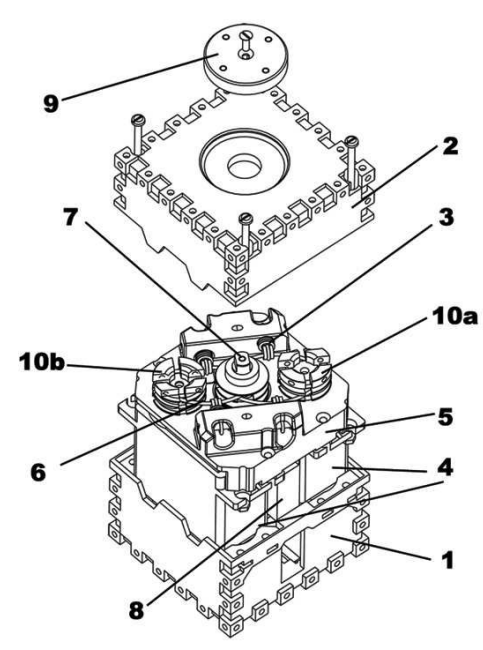

(a)

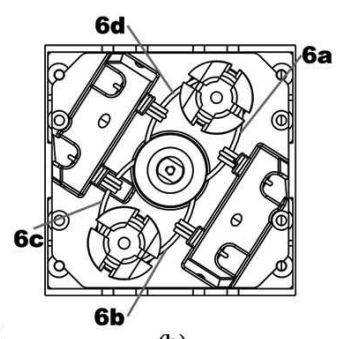

(b)

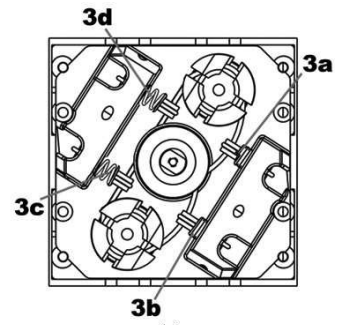

(c)
Fig. 5. Exploded 3D view of VSA-CUBE with basic components highlighted (a). Two configurations with different values of stiffness: minimum stiffness $(b)$ and maximum stiffness $(c)$ with no external loads.

\section{A. Mechanical design}

The actuator external shape is a cube with a $55 \mathrm{~mm}$ edge, (see Fig. 1,5). Small square grooves are machined along each edge to permit the interconnection of the unit with other components to form multi-DOF systems, as described in Sec.
IV. The external frame comprises two distinct components: a lower case (1) and an upper case (2). The main structural frame $(5)$ is rigidly connected to the lower frame (1). The two motor frames (4) are fixed on the lower face of component (5), so as to place the motor axes, on which two pulleys $(10 a, 10 b)$ are fixed, along the frame main diagonal. A bearing placed at the centre of the upper face of (5) supports the output shaft rotation (7). The elastic transmission is realized via four tendons $(6 a, 6 b, 6 c, 6 d)$ and four extension springs $(3 a, 3 b, 3 c, 3 d)$. One end of each tendon is wrapped and locked on to the output shaft, while the other end is wrapped and locked on to a pulley, as shown in Fig. 5(b). One end of each spring is locked on the upper face of (5) via a pin, while the other engages with a tendon. The output-shaft position sensor is placed on the backside of (5). An electronic driver board (8) is put inside the unit in the space left between the motors and provides connection to the electronic power and logic bus as in Fig. 3. Finally, an external flange (9) is fixed to the output shaft to actuate payloads.

The operation principle of a VSA is related to the layout adopted to implement the nonlinear spring, as described in [20]. The VSA-Cube uses a Bidirectional Antagonistic design, as shown in Fig. 5(b) and Fig. 5(c). This layout, already used in some actuators (e.g. [22]), is described and analysed in many papers (e.g. [21]).

Fig. 5(b) shows the system in a low stiffness configuration. In this situation springs (3) and tendons (6) are not loaded. When the two pulleys $(10 a)$ and $(10 b)$ rotate in opposite directions, two of the four tendons get loaded, stretching their springs and realising a configuration of high stiffness (see Fig. 5(c)). Movement of the output shaft (7), is simply realised by rotating the pulleys in the same direction. Some details about the mathematical characterisation and the stiffness function are reported in Sec. III.

To cut down the final cost, selection of material and components was constrained; all frames are made with ABS plastic using, for the first series of prototypes, rapid prototyping techniques. Pulleys and shafts are realized with aluminium and steel alloys. Motors are Hitec Inc. TH-7950 servo actuators (mechanical properties are shown in Fig. 2(b)), which use DC motors and linear steel gearboxes. Finally, tendons are made with Dynema fibres.

\section{B. Electronics and control interface}

From the electrical point of view, the system is actuated by two digital servos and the position of the output shaft is monitored by one potentiometer. Each unit is locally controlled by a Cypress microcontroller (CY8C27443-24PXI) which takes care of interpreting data from the potentiometer, controlling the motors and communicating with the external world. The electrical interface of every VSA-Cube is a fiveline bus, one for the ground, one for the power of the motors, one for the power of the logic and two implementing an $\mathrm{I}^{2} \mathrm{C}$ bus [23]. Multiple VSA-Cube units can be connected in series on the bus in a daisy-chain topology (see Fig. 6 for a logical scheme of the electronic, control and communication 


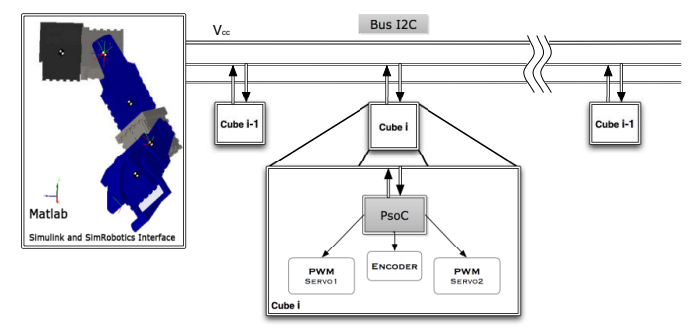

Fig. 6. Logical scheme of electronic and communication interfaces. Internal electronic of a cube is composed of a PSOC microcontroller that commands each servo via a PWM and can read output shaft position using a potentiometer. Each servo unit is connected to the main controller system via a $\mathrm{I}^{2} \mathrm{C}$ Bus.

interfaces). Each VSA-Cube unit has its own address on the bus. A writing operation on the unit has the effect of sending it a command, while a reading one is used to retrieve its internal configuration. Different control modalities are possible. The main one is servo-unit like: the rest position and the stiffness of the output shaft can be set. Other modalities include, but do not limit to, setting output shaft position (in closed loop as a non-VSA servo), and independent control of the two motors, but not limited to. This last option is made available to give the user the possibility to implement other low-level control strategies derived from the higher control layers.

\section{Mathematical Model}

A simple model of the VSA-Cube variable stiffness mechanism can be derived by the scheme of Fig. 7(a) (refer to caption for symbols definition). The function $\theta_{o s}=f(x)$,

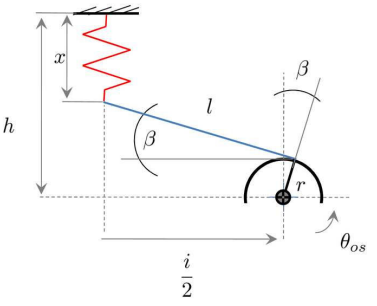

(a)

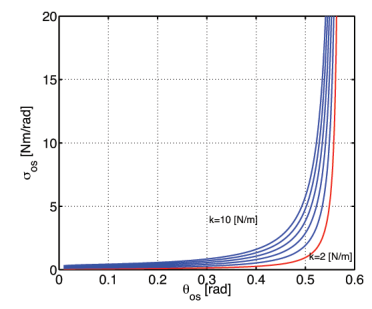

(b)
Fig. 7. Scheme of the stiffness regulation mechanical principle, $(a)$, and stiffness characteristic of the Cube VS mechanism, $(b)$. Symbols in panel (a) represent: $i$ the distance between centres of the pulleys; $l$ distance between free end of the spring and point in which the tendon is tangent to the pulley; $r$ radius of the pulley; $\beta$ the angle between $i$ and $l ; h$ the distance between fixed end of the spring and $i ; x$ the length of the spring; $\theta_{o s}$ the angular position of output shaft; $k$ is the spring elastic constant. Different lines on panel $(b)$ correspond to different values of the elastic constant of the springs, the red line matches the value of the springs adopted in the prototype.

describing how springs are loaded when the shaft or the pulleys rotate, can be obtained by solving the kinematics of the mechanism, described by

$$
\left\{\begin{array}{l}
\frac{i}{2}=l \cos (\beta)-r \sin (\beta) \\
h=x+l \sin (\beta)+r \cos (\beta) \\
\left(\theta_{o s}-\theta_{m, i}\right) r=2\left(l+r\left(\frac{\pi}{2}-\beta\right)\right)-(i+r \pi) .
\end{array}\right.
$$

Here $\theta_{m, i}$ represents angular position of the motor pulley, the output shaft is symmetrically placed with respect to spring axis. Output shaft stiffness can be evaluated by substituting $x_{i}$ with $f^{-1}\left(\theta_{o s}\right)$ in the equation

$$
\sigma_{o s}=\sum_{i=1}^{N_{s}} \frac{\partial^{2} U_{i}}{\partial \theta_{o s}^{2}}=\sum_{i=1}^{N_{s}} k_{i}\left(x_{i} \frac{\partial^{2} x_{i}}{\partial \theta_{o s}^{2}}+\left(\frac{\partial x_{i}}{\partial \theta_{o s}}\right)^{2}\right) .
$$

Since $f(\cdot)$ is not analytically invertible, the points of $f(x)$ were fitted with an approximating function (reported in caption of Fig. 8), with residuals smaller than 0.05 . We found the approximation robust with respect to the variation of the main design parameter $h$. Such approximation was used to
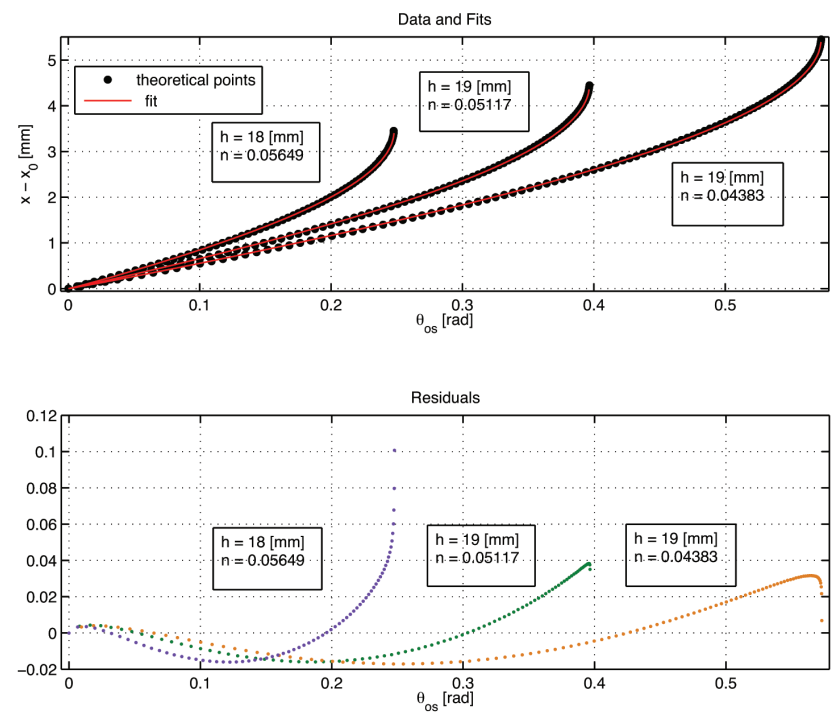

Fig. 8. Employed fitting curves of the stiffness characteristic. The function is $x=\hat{x}\left(\frac{2}{\pi} \arcsin \left(\frac{\theta_{o s}}{\hat{\theta}_{o s}}\right)\right)^{e^{n}}$, where $\hat{x}$ is the maximum value of $x$ and $\hat{\theta}_{O S}$ is corresponding value of $\theta_{O S}$. The value of $n$ is chosen in order to minimize the root mean square error. The chart in the lower part of the figure shows the residuals of the points of the three functions. Functions that are evaluated with three different value of $h$.

obtain the plot shown in Fig. 7(b). The torque exerted on output shaft $\tau_{o s}$ is the first derivative of elastic energy w.r.t. The output shaft angular position

$$
\tau_{o s}=\sum_{i=1}^{N_{s}} k_{i} x_{i} \frac{\partial x_{i}}{\partial \theta_{o s}}
$$

The working region of a VSA, on $(\sigma, \tau)$ plane, belongs to a bounded region. The two bounds can be found by considering the equilibrium conditions

$$
\tau_{o s}=\tau_{m, 1}+\tau_{m, 2},
$$

where $\tau_{m, 1}$ and $\tau_{m, 2}$ represent the torques of the motors.

The lower bound can be evaluated by assuming

$$
\tau_{m, 1}=\tau_{m, 2}=\frac{\tau_{o s}}{2},
$$

that for VSA-Cube corresponds to the minimum stiffness condition.

The upper bound is evaluated assuming the deformation of one side of the elastic transmission is maximized (for 
example this configuration is assumed by both sides of mechanism in Fig. 5(c)). This means that its stiffness value is maximum and the motor connected with this mechanism, e.g. motor one, supplies maximum torque:

$$
\tau_{m, 1}=\hat{\tau}_{m} .
$$

The torque supplied by the second motor, and by consequence the value of the stiffness, can be evaluated through Eq. 2.

\section{CUBE-BOT MULTIPLE DEGREES OF FREEDOM PLATFORM}

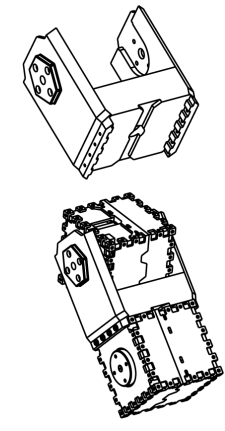

(a)
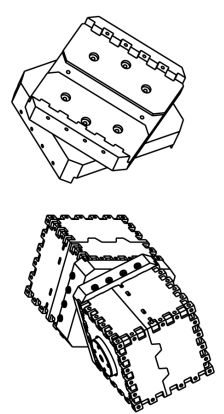

(b)

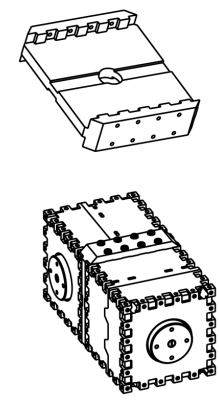

(c)
Fig. 9. The three possible types of connections that can be used to realize a robotics system. (a) and (b) show two kinds of revolute joints, with, respectively, perpendicular and parallel axis. $(c)$ shows the rigid connection.

Three basic connection possibilities make the VSA-Cube modules capable of forming different assemblies. Each connection is obtained by attaching a VSA-Cube with an interconnection flange, as shown in Fig. 9. Fig. 9(a) and 9(b) depict two kinds of revolute joints, with perpendicular and parallel axis respectively, while 9(c) shows a rigid connection. Fastening of the actuator unit on the flange is achieved by inserting the key of one actuator inside the flange's complementary groove. After this the connection is secured by using some screws. Fig. 10 shows four of the many possible assemblies that can be realised with the above connections, along with some other pieces not described here for brevity's sake. Fig. 10(a) shows a complete upperbody composed of a 1-DOF torso and two 5-DOF arms; Fig. 10(b) shows a pair of 6-DOF legs; Fig. 10(c) shows a eight-legged spider with three DOF per leg and Fig. 10(d) shows a 7-DOF arm, whose kinematic is inspired by the DLR-Light Weight Robot.

\section{A. Experiments}

A complete demostration of all the many possibilities of the VSA-CubeBot platform, is, by design, out of the space of a single paper. In fact as the quantity of available actuator units increases, the number of possible assemblies grows exponentially. An in-depth discussion on the control policies of multi-DOF variable stiffness robots is also out of the scope of this work. Both of these aspects are left open to future research. In this section we will just present

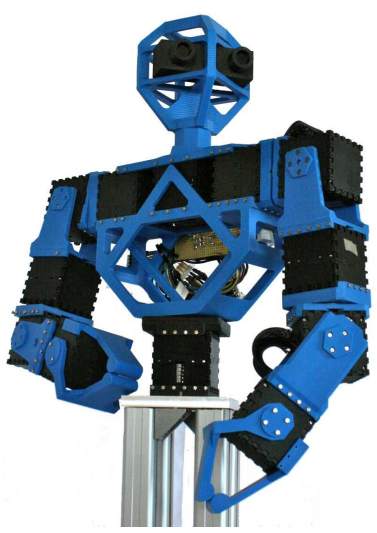

(a)

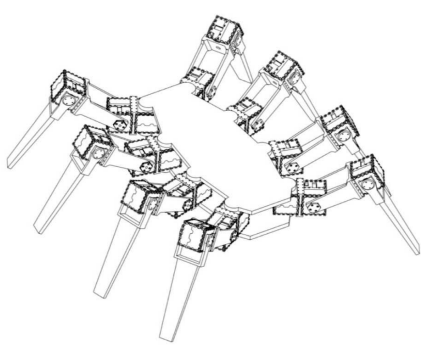

(c)

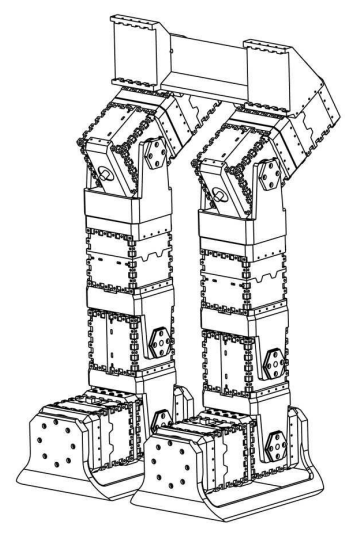

(b)

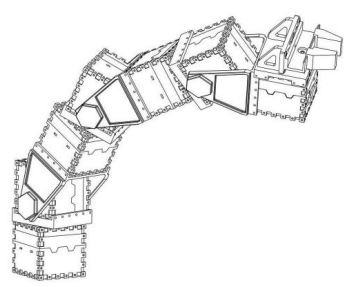

(d)
Fig. 10. Examples of possible robotic systems that can be built. In $(a)$ the realised upper body is shown, in $(b)$ a pair of 6 DOFs legs, in $(c)$ a a spider and in $(d)$ a seven degrees of freedom arm.

experimental results to demonstrate the performance of the VSA-Cube while regulating the stiffness. Five actuator units, and four interconnection elements, can be arranged as to form a simple arm with 4 DOFs of movement and a 1 DOF gripper. Its kinematic model is described by the Denavit Hartnberg parameters in Table I. A simple experiment has

\begin{tabular}{c|cccc}
$\mathrm{n}$ & $\alpha$ & $\mathrm{a}$ & $\theta$ & $\mathrm{d}$ \\
\hline 1 & $\pi / 2$ & 0 & $q_{1}-\pi / 2$ & 0 \\
2 & $-\pi / 2$ & 0 & $q_{2}$ & 0 \\
3 & $\pi / 2$ & 0 & $q_{3}$ & $d_{3}$ \\
4 & 0 & $a_{4}$ & $q_{4}-\pi / 2$ & 0 \\
\multicolumn{5}{|c}{ TABLE I }
\end{tabular}

DENAVIT-HARTENBERG TABLE OF THE 4 DOF ARM WITH A GRIPPER USED IN THE EXPERIMENTS.

been performed to show the stiffness variation of the cube.

The arm is posed in a vertical configuration and the shoulder module is actuated using a chirp reference signal for different stiffness pre-set angles. This way the arm oscillates around the shoulder axis.

Results shown in Fig.11, represent the experimental amplitude over frequency response. It is possible spot a clear resonance peak shifting to the high frequencies as the stiffness increases.

From Fig.11, with a reasonable approximation, it is possible to evaluate the stiffness of the shoulder module corresponding to the different preset at the corresponding resonance frequency. 
Given the inertia of the arm (w.r.t. the shoulder rotation axis), $0.052\left[\mathrm{kgm}^{2}\right]$, the stiffness range can be derived to be [6 - 12] $[\mathrm{Nm} / \mathrm{rad}]$. It is included in the theoretical range corresponding to the red curve in Fig. 7(b).

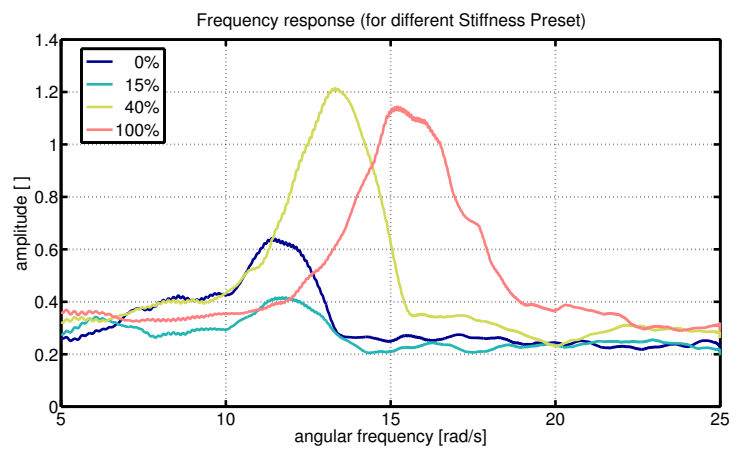

Fig. 11. Experimental amplitude frequency response of the arm oscillating: data is obtained feeding the shoulder first motor with a chirp signal. Different plots are realised for different stiffness preset values (see legend). It is possible to notice the shift of the resonant frequency corresponding to the stiffness increasing.

\section{CONCLUSIONS}

This paper present the VSA-Cubebot, a modular variable stiffness actuation unit suitable for multi dof variable stiffness robots. VSA-Cubebot is based on the low cost servo VSA units: the VSA Cubes. The mechanical and electrical details of a single unit were illustrated, along with the main performance parameters, experimentally validated, so as to provide a preliminary version of the actuator datasheet. Main interconnection details were illustrated, and some assembly ideas of robots realizable with the proposed hardware were shown. Experimental trials, demonstrating the functionality and behaviour of a simple 4-DOF arm realised with the VSACubeBot, are presented. There is a belief that the cost is one of the fundamental barriers which currently prevent the wide spread and experimentation of variable stiffness actuation. Our immediate future plans include the execution of a cost analysis study for producing the units in big quantities taking into account the various manufacturing possibilities. This phase will lead to the pre-production of a first batch of samples ready for the market.

\section{ACKNOWLEDGMENTS}

Authors would like to gratefully acknowledge the useful work done by Fabrizio Vivaldi, Andrea di Basco, Niccoló Capecci, Enrico Gastasini and Riccardo Schiavi. A special thanks goes to Giovanni Tonietti. This work was partially supported by the VIACTORS and THE Specific Targeted Research Projects, funded by the European Community under Contract IST-231554-2008 and ICT-248587-2010, respectively.

\section{REFERENCES}

[1] N. Hogan, "Impedance Control: An Approach to Manipulation: Part I, II and Ill," Journal of dynamic systems, measurement, and control, vol. 107 , p. $17,1985$.
[2] A. Albu-Schaffer, C. Ott, and G. Hirzinger, "A unified passivitybased control framework for position, torque and impedance control of flexible joint robots," The International Journal of Robotics Research, vol. 26, no. 1, p. 23, 2007.

[3] G. Tonietti, R. Schiavi, and A. Bicchi, "Design and control of a variable stiffness actuator for safe and fast physical human/robot interaction," in Proc. IEEE Int. Conf. on Robotics and Automation, 2005, pp. 528-533.

[4] M. Laffrachi, N. Tsagarakis, and D. Caldwell, "A variable physical damping actuator (VPDA) for compliant robotic joints," in Proc. IEEE International Conference on Robotics and Automation (ICRA), may. 2010, pp. $1668-1674$.

[5] A. Bicchi and G. Tonietti, "Fast and soft-arm tactics," IEEE Robotics and Automation Magazine, vol. 11, no. 2, pp. 22-33, 2004.

[6] J. Choi, S. Hong, W. Lee, S. Kang, and M. Kim, "A robot joint with variable stiffness using leaf springs," Robotics, IEEE Transactions on, vol. PP, no. 99, pp. $1-10,2011$.

[7] B. Vanderborght, R. Van Ham, D. Lefeber, T. G. Sugar, and K. W. Hollander, "Comparison of mechanical design and energy consumption of adaptable, passive-compliant actuators," Int. J. Rob. Res., vol. 28, no. 1, pp. 90-103, 2009.

[8] G. Palli, C. Melchiorri, and A. De Luca, "On the feedback linearization of robots with variable joint stiffness," in Proc. IEEE Int. Conf. on Robotics and Automation, 2008, pp. 1753-1759.

[9] M. G. Catalano, G. Grioli, F. Bonomo, R. Schiavi, and A. Bicchi, "Vsa-hd: From the enumeration analysis to the prototypical implementation," in EEE/RSJ International Conference on Intelligent RObots and Systems, Taipei, Taiwan, October 2010, accepted.

[10] Schunk official website. [Online]. Available: http://www.amtecrobotics.com/schunk/index.html?country=USA

[11] Harmonic drive official website. [Online]. Available: http://www.harmonicdrive.de/

[12] Bioloid official website. [Online]. Available: http://www.robotis.com

[13] Hitec official website. [Online]. Available: http://www.hitecrcd.com/

[14] Lego mindstorm official website. [Online]. Available: http://mindstorms.lego.com/en-us/Default.aspx

[15] Robocup mindstorm official website. [Online]. Available: http://www.robocup.org/

[16] E. Tira-Thompson, "Digital servo calibration and modeling," Robotics Institute, Pittsburgh, PA, Tech. Rep. CMU-RI-TR-09-41, March 2009.

[17] H. Lund and L. Pagliarini, "Robot soccer with lego mindstorms," in RoboCup-98: Robot Soccer World Cup II, ser. Lecture Notes in Computer Science, M. Asada and H. Kitano, Eds. Springer Berlin / Heidelberg, 2010, vol. 1604, pp. 141-151.

[18] J. Wolf, P. Hall, P. Robinson, and P. Culverhouse, "Bioloid based Humanoid Soccer Robot Design," in the Proc. of the Second Workshop on Humanoid Soccer Robots. Citeseer, 2007.

[19] M. Eaton, "Evolutionary humanoid robotics: past, present and future," 50 years of artificial intelligence: essays dedicated to the 50th anniversary of artificial intelligence, pp. 42-52, 2007.

[20] M. G. Catalano, R. Schiavi, and A. Bicchi, "Design of variable stiffness actuators mechanisms based on enumeration and analysis of performance," in IEEE International Conference on Robotics and Automation (ICRA2010), Anchorage, Alaska, May 3 - 8 2010, pp. $3285-3291$.

[21] F. Petit, M. Chalon, W. Friedl, M. Grebenstein, A. Schaffer, and G. Hirzinger, "Bidirectional antagonistic variable stiffness actuation: Analysis, design amp; implementation," in Robotics and Automation (ICRA), IEEE International Conference on, may. 2010, pp. 4189 4196.

[22] R. Schiavi, G. Grioli, S. Sen, and A. Bicchi, "Vsa-two: A novel prototype of variable stiffness actuator for safe and performing robots interacting with humans," in Proc. IEEE Int. Conf. on Robotics and Automation, 2008, pp. 2171 - 2176.

[23] I2c community website. [Online]. Available: http://www.i2c-bus.org/ 